\title{
Incidence and trends of childhood Type 1 diabetes worldwide 1990-1999
}

\author{
The DIAMOND Project Group
}

\section{Introduction}

The World Health Organization began the Multinational Project for Childhood Diabetes (DIAMOND) in 1990 [1]. Since then, standardized incidence data on Type 1 diabetes have been collected within the WHO DIAMOND Project until the year 1999.

The report on incidence of childhood Type 1 diabetes from 1990 to 1994 showed even larger global variation in incidence

Correspondence to: Marjatta Karvonen, National Public Health Institute, Mannerheimintie 166, SF-00300 Helsinki, Finland. E-mail: marjatta.karvonen@ktl.fi among children $\leq 14$ years of age than was reported previously in the 1970s and 1980s [2-7]. During the first half of the 1990s, the overall age-adjusted incidence of Type 1 diabetes varied from 0.1 in China and Venezuela to 37 per 100 000/ year in Sardinia and in Finland, representing a variation of over 350 -fold in the incidence in the 100 populations worldwide. The variation in the incidence of Type 1 diabetes seemed to reflect the global distribution of major ethnic populations which demonstrate a different degree of genetic susceptibility to diabetes. However, large differences in incidence have been reported from European populations living in relatively close proximity and in those who are genetically similar [8,9]. The rapid increase in the incidence of Type 1 diabetes has been almost a global phenomenon during the last decades $[8,10]$. 
In this study we investigated the age- and sex-specific incidence of Type 1 diabetes and trends in incidence between 1990 and 1999 worldwide.

\section{Materials and methods}

The detailed plan and organizational structure of the WHO DIAMOND incidence study have been described in previous reports $[1,2]$. Each of the participating centres is headed by a local principal investigator, who is responsible for data collection and the day-to-day aspects of the fieldwork (see Appendix). To be eligible to participate in the WHO DIAMOND study [1], each centre must have a well-defined population-based registry where the incidence can be accurately ascertained. Every centre prepared its own local methods of operation for the incidence study by following the framework provided by the WHO DIAMOND incidence study. In the local methods of operation, centres described the population base, the design of the registry, sources of data, data management, data items, and the time schedule for data collection. This analysis includes 112 centres from 57 countries. Most European countries included in the DIAMOND study are members of the EURODIAB Study Group [6].

\section{Incidence study population}

The total number of children aged 14 years or under living in the countries collaborating in the WHO DIAMOND incidence study was 740 million in 1996 . The denominator for the analyses which included the children $\leq 14$ years of age who resided in the study areas averaged 84 million per year. The numerator comprises 43013 children $\leq 14$ years of age diagnosed with Type 1 diabetes between the years 1990 and 1999 in the study areas.

\section{Classification and case definition}

The 1985 WHO classification of diabetes and the diagnostic criteria [11] are the basis of the minimum set of criteria for the WHO DIAMOND incidence study. Eligible individuals began daily insulin injections before their 15th birthday and were resident in the area of registration at the time of the first insulin administration. Registries are either prospective, retrospective or a combination of both. Participating centres have submitted annual incidence data to the WHO DIAMOND data centre in Helsinki using standardized forms. Data on gender, ethnic group, date of birth, date of first insulin administration, source of data (primary, secondary), family history of diabetes (the diabetic status of siblings, parents and children of registered cases) are collected. All the cases included in the database must have data at least on gender, the date of birth and the date of first insulin administration.

Completeness of registration was confirmed by estimating the degree of ascertainment using the capture-recapture method [12] in most centres. In some centres (see Fig. 1) this was not necessary as a result of complete coverage of the primary source. According to the WHO DIAMOND methods of operation, the primary data source consists of the cases of Type 1 diabetes who fulfil the criteria for registration and have been identified from hospital records, or from the records of paediatrician or family physician. Records of the local diabetes association, school health records or social insurance schemes have been used as a secondary (independent) source for cases.

\section{Statistical methods}

Incidence rates were calculated as the incidence per calendar year and 100000 population at risk without adjustment for under-reporting. Age adjustment for the rates was carried out using the direct method with a standard population consisting of equal numbers of children in each of the three age groups $(0-4$, $5-9,10-14$ years of age). The $95 \%$ confidence intervals were estimated assuming the Poisson distribution of the cases. The incidence rates were divided into five groups, as in our earlier report: (i) very low, < 1 per 100 000/year; (ii) low, 1-4.99 per 100 000/year; (iii) intermediate, 5-9.99 per 100 000/year; (iv) high, 10-19.99 per $100000 /$ year, and (v) very high, $\geq 20$ per 100 000/year [2].

Poisson regression models were used to analyse the incidence rates by centre, gender, age, and calendar year, and their interactions. Models were fitted using the S-PLUS.

\section{Results}

\section{Incidence of Type 1 diabetes}

During the period 1990-1999, the overall age-adjusted incidence of Type 1 diabetes in 112 centres (114 populations) varied from 0.1/100 000/year in China and Venezuela to 40.9 per 100 000/year in Finland (Table 1, Fig. 1). In most Asian populations the incidence was very low; $70 \%$ of them having an incidence of < 1 per 100 000/year. A marked exception among the Asian populations was Kuwait, with a very high incidence of 22 per 100 000/year. Among African populations, incidence was low or intermediate, ranging between 1 per 100 000/year and 9 per 100 000/year. The incidence among South American populations varied between very low to high, < 1 per 100 000/year to 10 per 100 000/year. In Central America and the West Indies, the range of variation was from 2 per 100 000/year to 17 per 100 000/year. The highest incidence rates were among European and North American populations varying from low, 4 per 100 000/year, to very high, 41 per 100 00/year, in Europe and from high, 11 per 100 000/year, to very high, 25 per 100 000/year, in North America. In Oceania, the incidence of Type 1 diabetes was also high or very high, ranging from 14 per 100 000/year to 22 per 100 000/year, reflecting difference in the ethnicity of populations within this region. There was also a marked within-country variation in the ageadjusted incidence in most of countries having data from at least two centres (Table 1).

\section{Age and sex differences in incidence}

The age-specific incidence rates were calculated for three age groups (0-4, 5-9 and 10-14 years) separately for boys and 
Figure 1 Age-standardized incidence of Type 1 diabetes in children under 14 years of age (per 100000 per year). Countries are arranged in descending order according to the incidence.

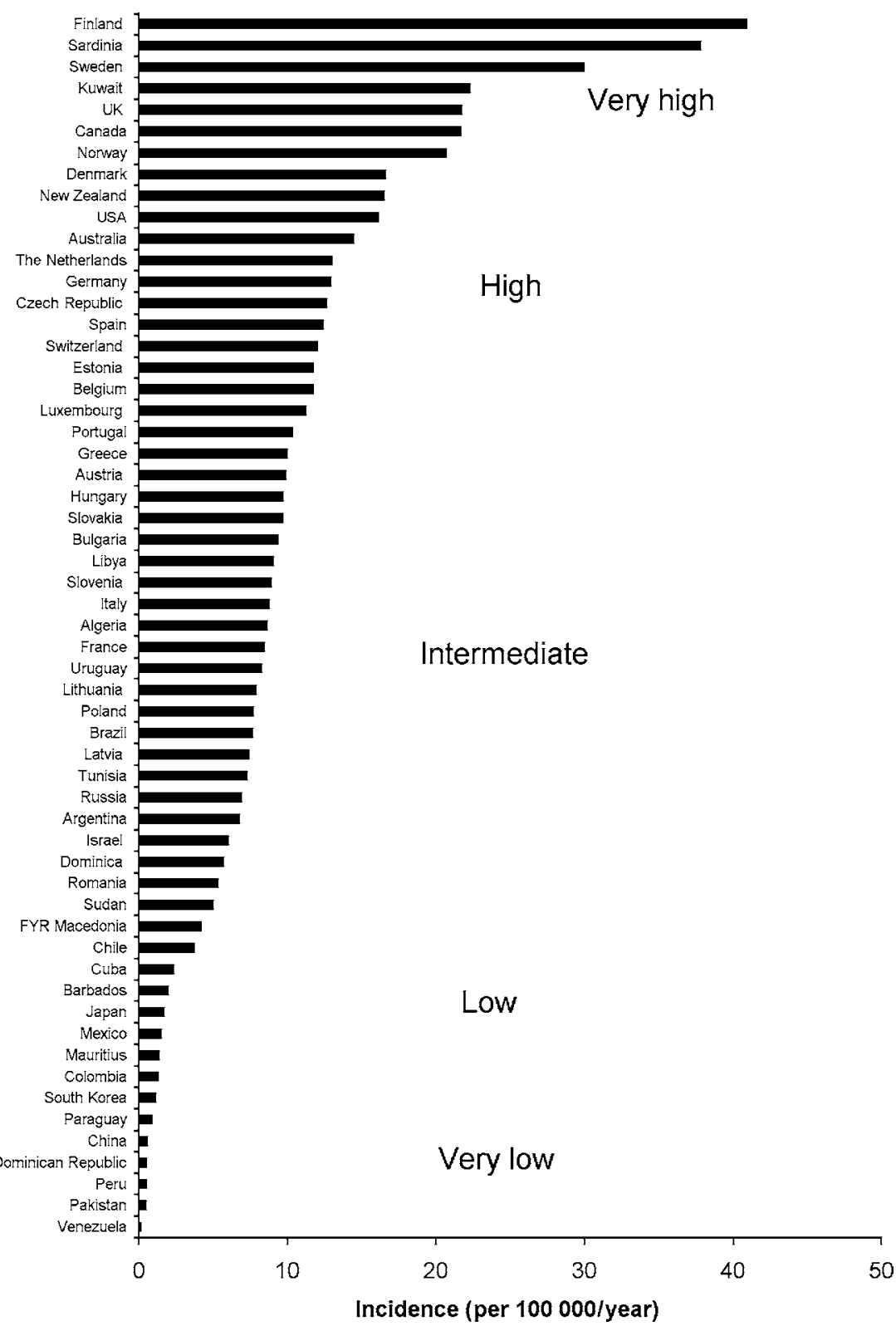

girls On the whole, there were no marked differences in agegroup specific incidence between the genders. The differences in incidence rates between age groups were statistically significant $(P<0.001)$ in almost all countries. In general, the incidence increased with age (Table 2 ). In the pooled data, the 5-9-year olds had 1.62 (1.57-1.66) times higher risk, and the 10-14-year olds 1.94 (1.89-1.98) times higher risk than that of the 0-4-year olds. The effect of age on the incidence did not differ between the genders. The exceptions were two countries of very high incidence, namely Finland and Sweden, and two countries with intermediate incidence, i.e. Romania and Lithuania. In all these countries, the age-specific incidence rates increased from 0 to 4 years to $10-14$ years among boys, whereas among girls incidence increased only from the age group $0-4$ years to the ages 5-9 years.

\section{The global trend in the incidence of Type 1 diabetes}

The mean annual increase in incidence calculated from 103 centres (submitted data for 3 years or more) was $2.8 \%$ (95\% CI 2.4-3.2\%; Table 3). In the first 5-year study from 1990 to 1994 , the average annual increase was $2.4 \%$ (1.3-3.4\%). During the second study period from 1995 to 1999 , the annual increase was higher at $3.4 \%(2.7-4.3 \%)$, but the difference in the trend between periods was not statistically significant.

The trend in incidence did not differ significantly between genders, except in Kuwait, Bulgaria, and Cuba. In Kuwait, the annual increase was $2.9 \%(-2.4$ to $8.6 \%)$ in boys and $11.2 \%$ $(5.4-17.4 \%)$ in girls, in Bulgaria there was an increase of $7.5 \%$ $(4.2-10.8 \%)$ in boys and an increase of $2.4 \%(-0.9$ to $5.9 \%)$ 
Table 1 Age-standardized incidence of Type 1 diabetes in children aged 14 years or under (per 100 000/year)

\begin{tabular}{|c|c|c|c|c|c|c|c|c|c|}
\hline \multirow[b]{2}{*}{ Region Country and area } & \multirow[b]{2}{*}{ Study period } & \multirow{2}{*}{$\begin{array}{l}\text { Estimate of } \\
\text { ascertainment \% }\end{array}$} & \multicolumn{4}{|c|}{ Incidence } & \multirow[b]{2}{*}{ Boys/girls } & \multicolumn{2}{|c|}{$\begin{array}{l}\text { Annual change } \\
\text { of incidence }\end{array}$} \\
\hline & & & Boys & Girls & Total & $(95 \% \mathrm{CI})$ & & $\%$ & $(95 \% \mathrm{CI})$ \\
\hline Africa & & & & & & & & 3.0 & $(0.3 ; 5.8)$ \\
\hline Algeria & & & & & & & & 11.6 & $(5.5 ; 18.0)$ \\
\hline Oran* & 1990-1999 & & 7.7 & 9.6 & 8.6 & $(7.6 ; 9.8)$ & 0.8 & & \\
\hline Libya & & & & & & & & -0.9 & $(-5.6 ; 4.0)$ \\
\hline Benghazi & 1991-1999 & & 7.8 & 10.3 & 9.0 & $(8.0 ; 10.2)$ & 0.8 & & \\
\hline Mauritius & 1990-1994 & $35-100$ & 1.2 & 1.4 & 1.3 & $(0.8 ; 2.0)$ & 0.9 & -2.2 & $(-27.8 ; 32.3)$ \\
\hline \multicolumn{10}{|l|}{ Sudan } \\
\hline Gezira & 1990 & 100 & 5.6 & 4.4 & 5.0 & $(3.8 ; 6.5)$ & 1.3 & & \\
\hline Tunisia & & & & & & & & 0.7 & $(-3.5 ; 5.2)$ \\
\hline Beja* & 1990-1999 & & 8.4 & 6.9 & 7.7 & $(6.1 ; 9.6)$ & 1.2 & & \\
\hline Gafsa* & 1990-1999 & & 9.5 & 7.5 & 8.5 & $(6.9 ; 10.3)$ & 1.3 & & \\
\hline Kairoan* & 1991-1993 & & 7.3 & 7.8 & 7.6 & $(5.6 ; 10.0)$ & 0.9 & & \\
\hline Monastir* & 1990-1999 & & 6.6 & 5.1 & 5.8 & $(4.6 ; 7.3)$ & 1.3 & & \\
\hline Asia & & & & & & & & 4.0 & $(1.8 ; 6.2)$ \\
\hline China & & & & & & & & -0.1 & $(-5.7 ; 5.8)$ \\
\hline Beijing* & 1990-1994 & & 0.7 & 1.1 & 0.9 & $(0.7 ; 1.1)$ & $0.6 \dagger$ & & \\
\hline Chang Chun & 1990-1994 & $86-100$ & 0.6 & 1.1 & 0.8 & $(0.5 ; 1.3)$ & 0.6 & & \\
\hline Changsha & 1990-1994 & 100 & 0.2 & 0.2 & 0.2 & $(0.2 ; 0.4)$ & 1.3 & & \\
\hline Dalian & 1990-1994 & 100 & 1.1 & 1.2 & 1.2 & $(0.7 ; 1.7)$ & 0.9 & & \\
\hline Guilin & 1991-1994 & 100 & 0.6 & 1.0 & 0.8 & $(0.2 ; 2.0)$ & 0.6 & & \\
\hline Hainan & 1990-1994 & 100 & 0.1 & 0.2 & 0.2 & $(0.1 ; 0.2)$ & 0.5 & & \\
\hline Harbin & 1990-1996 & 100 & 0.6 & 0.6 & 0.6 & $(0.4 ; 0.8)$ & 1.0 & & \\
\hline Hong-Kong* & 1990-1995 & & 0.6 & 1.9 & 1.3 & $(0.8 ; 1.9)$ & $0.3 \dagger$ & & \\
\hline Huhehot & 1990-1994 & 100 & 1.1 & 0.7 & 0.9 & $(0.5 ; 1.5)$ & 1.5 & & \\
\hline Jilin & 1990-1994 & 100 & 0.4 & 0.8 & 0.6 & $(0.4 ; 0.9)$ & 0.5 & & \\
\hline Jinan & 1990-1995 & 100 & 0.5 & 0.4 & 0.4 & $(0.3 ; 0.6)$ & 1.1 & & \\
\hline Lanzhou & 1991-1994 & 100 & 0.4 & 0.2 & 0.3 & $(0.1 ; 0.5)$ & 1.9 & & \\
\hline Nanjing & 1990-1995 & 100 & 0.5 & 1.2 & 0.9 & $(0.6 ; 1.2)$ & 0.5 & & \\
\hline Nanning & 1990-1994 & 100 & 0.3 & 0.7 & 0.5 & $(0.3 ; 0.8)$ & 0.4 & & \\
\hline Shanghai & 1990-1994 & 69-100 & 0.7 & 0.7 & 0.7 & $(0.5 ; 0.9)$ & 0.9 & & \\
\hline Shenyang & 1990-1994 & 100 & 0.4 & 0.5 & 0.5 & $(0.3 ; 0.7)$ & 0.9 & & \\
\hline Sichuan & 1990-1994 & $80-100$ & 1.8 & 2.7 & 2.3 & $(1.4 ; 3.3)$ & 0.7 & & \\
\hline Tie Ling & 1990-1994 & 100 & 0.2 & 0.1 & 0.2 & $(0.1 ; 0.3)$ & 1.0 & & \\
\hline Wuhan & 1990-1994 & 100 & 5.2 & 3.8 & 4.5 & $(2.8 ; 7.0)$ & 1.5 & & \\
\hline Wulumuqi & 1990-1994 & 100 & 0.9 & 0.8 & 0.8 & $(0.3 ; 1.7)$ & 1.2 & & \\
\hline Zhengzhou & 1991-1994 & $86-100$ & 0.2 & 1.0 & 0.6 & $(0.3 ; 1.1)$ & 0.2 & & \\
\hline Zunyi & 1990-1995 & 100 & 0.0 & 0.1 & 0.1 & $(0.0 ; 0.2)$ & 0.6 & & \\
\hline Israel $\neq$ & 1990-1993 & 100 & 5.5 & 6.6 & 6.0 & $(5.4 ; 6.7)$ & $0.8 \dagger$ & 7.6 & $(-2.0 ; 18.1)$ \\
\hline Japan & & & & & & & & -3.5 & $(-15.7 ; 10.6)$ \\
\hline Chiba* & 1990-1993 & & 1.2 & 1.6 & 1.4 & $(1.1 ; 1.8)$ & 0.8 & & \\
\hline Hokkaido & 1990-1993 & 100 & 2.2 & 2.1 & 2.2 & $(1.7 ; 2.6)$ & 1.0 & & \\
\hline Okinawa & 1990-1993 & $77-100$ & 1.0 & 1.8 & 1.4 & $(0.8 ; 2.2)$ & 0.6 & & \\
\hline Kuwait & 1992-1999 & $79-96$ & 21.7 & 22.9 & 22.3 & $(20.5 ; 24.2)$ & 1.0 & 7.0 & $(3.0 ; 11.1)$ \\
\hline Pakistan & & & & & & & & -5.6 & $(-11.2 ; 0.3)$ \\
\hline Karachi & 1990-1999 & 51 & 0.4 & 0.5 & 0.5 & $(0.3 ; 0.5)$ & 0.8 & & \\
\hline Russia & & & & & & & & 6.6 & $(3.0 ; 10.3)$ \\
\hline Novosibirsk & 1990-1999 & $87-100$ & 6.8 & 7.1 & 6.9 & $(6.3 ; 7.6)$ & 1.0 & & \\
\hline South Korea & & & & & & & & & \\
\hline Seoul $*$ & 1990-1991 & & 1.1 & 1.2 & 1.1 & $(0.9 ; 1.5)$ & 0.9 & & \\
\hline Europe & & & & & & & & 3.2 & $(2.7 ; 3.6)$ \\
\hline Austria $\ddagger$ & 1990-1999 & 99-100 & 10.3 & 9.5 & 9.9 & $(9.4 ; 10.4)$ & 1.1 & 2.1 & $(-0.4 ; 4.8)$ \\
\hline Belgium $\ddagger$ & & & & & & & & 1.5 & $(-3.4 ; 6.7)$ \\
\hline Antwerpen & 1990-1999 & 90-100 & 10.7 & 12.8 & 11.7 & $(10.2 ; 13.5)$ & 0.8 & & \\
\hline Bulgaria & & & & & & & & 5.1 & $(2.8 ; 7.5)$ \\
\hline Varna & 1990-1999 & 100 & 7.9 & 8.3 & 8.1 & $(7.4 ; 9.0)$ & 1.0 & & \\
\hline West-Bulgaria & 1990-1999 & $99-100$ & 11.6 & 9.8 & 10.7 & $(9.8 ; 11.6)$ & $1.2 \dagger$ & & \\
\hline Czech Republic $\ddagger$ & 1995-1999 & & 12.6 & 12.7 & 12.7 & $(11.9 ; 13.5)$ & 1.0 & 9.6 & $(5.2 ; 14.2)$ \\
\hline
\end{tabular}


Table 1 Continued

\begin{tabular}{|c|c|c|c|c|c|c|c|c|c|}
\hline \multirow[b]{2}{*}{ Region Country and area } & \multirow[b]{2}{*}{ Study period } & \multirow{2}{*}{$\begin{array}{l}\text { Estimate of } \\
\text { ascertainment \% }\end{array}$} & \multicolumn{4}{|c|}{ Incidence } & \multirow[b]{2}{*}{ Boys/girls } & \multicolumn{2}{|c|}{$\begin{array}{l}\text { Annual change } \\
\text { of incidence }\end{array}$} \\
\hline & & & Boys & Girls & Total & $(95 \% \mathrm{CI})$ & & $\%$ & $(95 \% \mathrm{CI})$ \\
\hline Denmark $\ddagger$ & & & & & & & & 15 & $(-2.6 ; 5.7)$ \\
\hline Four counties & 1990-1999 & $83-100$ & 17.1 & 16.2 & 16.6 & $(14.9 ; 18.4)$ & 1.1 & & \\
\hline Estonia* & 1990-1999 & & 12.6 & 10.9 & 11.7 & $(10.6 ; 13.0)$ & 1.1 & 3.7 & $(0.1 ; 7.5)$ \\
\hline Finland* & 1990-1999 & & 41.9 & 39.9 & 40.9 & $(39.6 ; 42.2)$ & $1.1 \dagger$ & 4.2 & $(3.1 ; 5.3)$ \\
\hline France $\neq$ & & & & & & & & 4.8 & $(-0.5 ; 10.5)$ \\
\hline Four regions & 1990-1994 & $95-99$ & 8.7 & 8.3 & 8.5 & $(7.9 ; 9.1)$ & 1.1 & & \\
\hline FYR Macedoniał & 1995-1999 & & 4.9 & 3.5 & 4.2 & $(3.4 ; 5.2)$ & 1.4 & 9.7 & $(-9.6 ; 33.2)$ \\
\hline Germany & & & & & & & & 2.3 & $(0.8 ; 3.8)$ \\
\hline Düsseldorf & 1995-1999 & $97-98$ & 14.8 & 16.1 & 15.4 & $(13.8 ; 17.2)$ & 0.9 & & \\
\hline Baden-Württemberg & 1990-1999 & $91-100$ & 12.7 & 12.6 & 12.6 & $(12.1 ; 13.2)$ & 1.0 & & \\
\hline Greeceł & & & & & & & & 0.9 & $(-2.0 ; 3.8)$ \\
\hline Attica & 1990-1999 & 100 & 11.0 & 9.0 & 10.0 & $(9.2 ; 10.9)$ & $1.2 \dagger$ & & \\
\hline Hungary $\ddagger$ & & & & & & & & 2.6 & $(0.8 ; 4.5)$ \\
\hline Eighteen counties & 1990-1999 & $99-100$ & 9.6 & 9.8 & 9.7 & $(9.2 ; 10.2)$ & 1.0 & & \\
\hline Italy & & & & & & & & $0.9 \mathbb{S}$ & $(-0.9 ; 2.7)$ \\
\hline Lazioł* & 1990-1999 & & 8.9 & 8.6 & 8.8 & $(8.1 ; 9.4)$ & 1.0 & & \\
\hline Lombardiał & 1990-1995 & 100 & 7.2 & 6.5 & 6.9 & $(6.3 ; 7.5)$ & 1.1 & & \\
\hline Marche & 1990-1999 & 100 & 10.5 & 9.7 & 10.1 & $(8.8 ; 11.6)$ & 1.1 & & \\
\hline Pavia & 1990-1999 & 100 & 12.3 & 12.5 & 12.4 & $(9.7 ; 15.6)$ & 1.0 & & \\
\hline Sardiniał & 1990-1998 & $37-85$ & 45.0 & 30.6 & 37.8 & $(35.5 ; 40.3)$ & 1.5 & 1.4 & $(-1.1 ; 3.9)$ \\
\hline Eastern Sicily $\ddagger$ & 1990-1994 & $96-100$ & 13.4 & 9.9 & 11.7 & $(9.8 ; 13.9)$ & 1.3 & & \\
\hline Turin & 1990-1999 & $97-100$ & 11.7 & 10.3 & 11.0 & $(9.8 ; 12.3)$ & 1.1 & & \\
\hline Latvia & 1990-1999 & & 7.8 & 7.0 & 7.4 & $(6.6 ; 8.3)$ & 1.1 & 3.1 & $(-0.6 ; 6.8)$ \\
\hline Lithuania & 1990-1999 & 100 & 7.6 & 8.2 & 7.9 & $(7.3 ; 8.5)$ & 0.9 & 2.5 & $(-0.2 ; 5.3)$ \\
\hline Luxemburg $\ddagger$ & 1990-1999 & 100 & 10.3 & 12.2 & 11.3 & $(9.0 ; 13.9)$ & 0.8 & -0.1 & $(-7.2 ; 8.1)$ \\
\hline The Netherlands $\ddagger$ & & & & & & & & 3.4 & $(-4.0 ; 11.3)$ \\
\hline Five regions & 1990-1994 & $87-98$ & 12.9 & 13.2 & 13.0 & $(11.7 ; 14.4)$ & 1.0 & & \\
\hline Norway $\ddagger$ & & & & & & & & -0.9 & $(-3.2 ; 1.4)$ \\
\hline Eight counties & 1990-1999 & $91-100$ & 21.6 & 19.9 & 20.8 & $(19.4 ; 22.1)$ & 1.1 & & \\
\hline Poland & & & & & & & & 7.6 & $(4.9 ; 10.4)$ \\
\hline Cracow* & 1990-1999 & 100 & 7.5 & 7.6 & 7.6 & $(7.0 ; 8.2)$ & 1.0 & & \\
\hline Upper Silesia $\ddagger$ & 1995-1999 & & 8.0 & 9.5 & 8.8 & $(7.9 ; 9.7)$ & 0.8 & & \\
\hline Wielkopolska & 1990 & 100 & 4.1 & 6.0 & 5.0 & $(3.9 ; 6.4)$ & 0.7 & & \\
\hline Portugal & & & & & & & & 2.0 & $(-4.1 ; 8.4)$ \\
\hline Algarveł & 1990-1994 & $74-100$ & 16.3 & 12.9 & 14.6 & $(10.6 ; 19.6)$ & 1.3 & & \\
\hline Coimbra & 1990-1999 & 100 & 10.1 & 9.1 & 9.6 & $(7.6 ; 12.2)$ & 1.1 & & \\
\hline Madeira Island $\ddagger$ & 1990-1999 & 100 & 7.1 & 6.8 & 6.9 & $(5.0 ; 9.4)$ & 1.1 & & \\
\hline Portalegreł & 1990-1994 & $86-100$ & 15.9 & 26.7 & 21.3 & $(13.3 ; 31.9)$ & 0.6 & & \\
\hline Romania & & & & & & & & 2.8 & $(-3.9 ; 10.0)$ \\
\hline Bucharest & 1990-1999 & 100 & 4.7 & 5.9 & 5.3 & $(4.7 ; 6.1)$ & 0.8 & & \\
\hline Slovakia & 1990-1999 & 100 & 9.7 & 9.7 & 9.7 & $(9.2 ; 10.3)$ & 1.0 & 6.3 & $(4.3 ; 8.5)$ \\
\hline Sloveniał & 1990-1999 & 100 & 8.3 & 9.5 & 8.9 & $(8.0 ; 9.9)$ & 0.9 & 3.3 & $(-0.5 ; 7.2)$ \\
\hline Spain & & & & & & & & -1.9 & $(-3.7 ; 0.0)$ \\
\hline Catalonia & 1990-1999 & $50-96$ & 12.6 & 12.3 & 12.4 & $(11.7 ; 13.1)$ & 1.0 & & \\
\hline Sweden* & 1990-1999 & 100 & 30.5 & 29.4 & 30.0 & $(29.1 ; 30.8)$ & 1.0 & 3.6 & $(2.6 ; 4.7)$ \\
\hline Switzerland $\ddagger$ & 1995-1999 & & 13.3 & 10.7 & 12.0 & $(11.2 ; 12.9)$ & $1.2 \dagger$ & 2.1 & $(-3.1 ; 7.5)$ \\
\hline UK & & & & & & & & 4.0 & $(3.0 ; 5.0)$ \\
\hline Scotland (Aberdeen) & 1990-1999 & 100 & 26.8 & 25.9 & 26.4 & $(25.4 ; 27.4)$ & 1.0 & & \\
\hline Leicestershire $\ddagger$ & 1990-1994 & $97-100$ & 15.4 & 15.3 & 15.3 & $(12.9 ; 18.1)$ & 1.0 & & \\
\hline Northern Ireland $\ddagger$ & 1990-1999 & $95-100$ & 21.5 & 21.2 & 21.3 & $(19.9 ; 22.8)$ & 1.0 & & \\
\hline Oxford $\neq *$ & 1990-1994 & & 20.1 & 15.3 & 17.7 & $(16.2 ; 19.5)$ & $1.3+$ & & \\
\hline Plymouth & 1990-1999 & $96-100$ & 17.1 & 20.8 & 19.0 & $(16.8 ; 21.2)$ & 0.9 & & \\
\hline Yorkshire‡ & 1990-1999 & 99 & 18.9 & 18.1 & 18.5 & $(17.5 ; 19.5)$ & 1.0 & & \\
\hline North America & & & & & & & & 5.3 & $(3.3 ; 7.3)$ \\
\hline Canada & & & & & & & & 5.1 & $(1.9 ; 8.5)$ \\
\hline Edmonton & 1990-1996 & $75-96$ & 23.0 & 23.6 & 23.3 & $(20.5 ; 26.4)$ & 1.0 & & \\
\hline Calgary & 1990-1999 & 100 & 20.3 & 20.9 & 20.6 & $(18.5 ; 22.7)$ & 1.0 & & \\
\hline Prince Edward Island* & 1990-1993 & 100 & 28.0 & 20.8 & 24.5 & $(16.4 ; 35.2)$ & 1.3 & & \\
\hline
\end{tabular}




\begin{tabular}{|c|c|c|c|c|c|c|c|c|c|}
\hline \multirow[b]{2}{*}{ Region Country and area } & \multirow[b]{2}{*}{ Study period } & \multirow{2}{*}{$\begin{array}{l}\text { Estimate of } \\
\text { ascertainment \% }\end{array}$} & \multicolumn{4}{|c|}{ Incidence } & \multirow[b]{2}{*}{ Boys/girls } & \multicolumn{2}{|c|}{$\begin{array}{l}\text { Annual change } \\
\text { of incidence }\end{array}$} \\
\hline & & & Boys & Girls & Total & $(95 \% \mathrm{CI})$ & & $\%$ & $(95 \% \mathrm{CI})$ \\
\hline USA & & & & & & & & 5.5 & $(3.0 ; 8.0)$ \\
\hline Allegheny, PA & 1990-1994 & $87-100$ & 19.1 & 16.4 & 17.8 & $(15.5 ; 20.3)$ & 1.2 & & \\
\hline \multicolumn{10}{|l|}{ Chicago, IL } \\
\hline African-American & 1990-1999 & $51-100$ & 15.2 & 19.4 & 17.3 & $(15.8 ; 18.7)$ & $0.8+$ & & \\
\hline Hispanic & 1990-1999 & $51-100$ & 11.7 & 11.1 & 11.4 & $(10.1 ; 12.9)$ & 1.1 & & \\
\hline Others & 1995-1999 & $>75$ & 19.0 & 17.5 & 18.3 & $(15.7 ; 22.2)$ & 1.1 & & \\
\hline Jefferson, $\mathrm{AL}^{*}$ & 1990-1995 & & 14.1 & 15.1 & 14.6 & $(12.2 ; 18.2)$ & 0.9 & & \\
\hline South America & & & & & & & & 5.3 & $(2.8 ; 7.9)$ \\
\hline Argentina & & & & & & & & 0.4 & $(-8.8 ; 10.5)$ \\
\hline Avellaneda & 1990-1996 & $88-100$ & 5.3 & 7.2 & 6.3 & $(5.7 ; 11.1)$ & 0.7 & & \\
\hline Cordoba & 1991-1992 & $88-92$ & 6.2 & 7.9 & 7.0 & $(5.2 ; 9.3)$ & 0.8 & & \\
\hline Corrientes & 1992-1999 & $90-100$ & 4.7 & 8.5 & 6.6 & $(5.0 ; 8.7)$ & 0.6 & & \\
\hline Tierra del Fuego & 1993-1996 & 100 & 14.2 & 6.3 & 10.3 & $(5.5 ; 18.5)$ & 2.2 & & \\
\hline Brazil & & & & & & & & -16.0 & $(-48.6 ; 37.2)$ \\
\hline Sao Paulo & 1990-1992 & $70-95$ & 6.9 & 9.1 & 8.0 & $(5.5 ; 11.1)$ & 0.8 & & \\
\hline Passo Fundo & 1996-1999 & 100 & 5.4 & 8.7 & 7.0 & $(4.1 ; 11.9)$ & 0.6 & & \\
\hline Chile & & & & & & & & 7.5 & $(4.3 ; 10.9)$ \\
\hline Santiago & 1990-1999 & 100 & 3.6 & 3.9 & 3.7 & $(3.4 ; 4.0)$ & 0.9 & & \\
\hline \multicolumn{10}{|l|}{ Colombia } \\
\hline Cali* & 1995-1999 & & 0.4 & 0.5 & 0.5 & $(0.3 ; 0.7)$ & 0.8 & & \\
\hline Bogota & 1990 & 97 & 4.7 & 2.9 & 3.8 & $(2.9 ; 4.9)$ & 1.6 & & \\
\hline Paraguay* & 1990-1999 & & 1.0 & 0.8 & 0.9 & $(0.8 ; 1.0)$ & 1.1 & -0.5 & $(-5.7 ; 4.9)$ \\
\hline Peru & & & & & & & & 12.1 & $(-7.5 ; 35.8)$ \\
\hline Lima & 1990-1994 & $35-100$ & 0.4 & 0.6 & 0.5 & $(0.4 ; 0.64)$ & 0.7 & & \\
\hline \multicolumn{10}{|l|}{ Uruguay } \\
\hline Montevideo & 1992 & 97 & 8.3 & 8.2 & 8.3 & $(5.4 ; 11.7)$ & 1.0 & & \\
\hline Venezuela & & & & & & & & -6.8 & $(-24.6 ; 15.3)$ \\
\hline Caracas* & 1990-1994 & & 0.1 & 0.2 & 0.1 & $(0.1 ; 0.2)$ & 0.7 & & \\
\hline Central America and West & & & & & & & & -3.6 & $(-5.0 ;-2.2)$ \\
\hline \multicolumn{10}{|l|}{ Indies } \\
\hline Barbados* & 1990-1993 & & 2.4 & 1.6 & 2.0 & $(0.6 ; 4.1)$ & 1.5 & & \\
\hline Cuba & 1990-1999 & $25-100$ & 2.1 & 2.5 & 2.3 & $(2.2 ; 2.5)$ & 0.8 & -10.8 & $(-13.4 ;-8.2)$ \\
\hline Dominica & 1990-1993 & & 6.6 & 4.9 & 5.7 & $(2.4 ; 12.6)$ & 1.3 & -46.1 & $(-78.3 ; 34.0)$ \\
\hline Dominican Republic & 1995-1999 & $39-67$ & 0.7 & 0.3 & 0.5 & $(0.4 ; 0.7)$ & $2.3 \dagger$ & 12.6 & $(-11.4 ; 43.0)$ \\
\hline \multicolumn{10}{|l|}{ Mexico } \\
\hline Veracruz & 1990-1993 & 100 & & & 1.5 & $(0.7 ; 2.9)$ & & & \\
\hline Puerto Rico (USA) & 1990-1999 & $90-97$ & 15.8 & 17.8 & 16.8 & $(16.0 ; 17.6)$ & $0.9 \dagger$ & -1.0 & $(-2.7 ; 0.7)$ \\
\hline Virgin Islands (USA)* & 1990-1996 & & & 14.0 & 12.8 & $(8.1 ; 18.8)$ & & 7.1 & $(-13.2 ; 32.1)$ \\
\hline Oceania & & & & & & & & 3.2 & $(-0.4 ; 6.9)$ \\
\hline Australia & & & & & & & & 4.1 & $(-2.5 ; 11.1)$ \\
\hline New South Wales & 1990-1993 & $89-100$ & 13.1 & 15.9 & 14.5 & $(13.5 ; 15.6)$ & $0.8 \dagger$ & & \\
\hline New Zealand & & & & & & & & 2.8 & $(-1.4 ; 7.2)$ \\
\hline Auckland & 1990-1996 & 100 & 12.9 & 14.6 & 13.7 & $(12.0 ; 15.7)$ & 0.9 & & \\
\hline Canterbury & 1990-1999 & 100 & 23.8 & 20.8 & 22.3 & $(19.1 ; 25.9)$ & 1.1 & & \\
\hline
\end{tabular}

*One source.

†Statistically significant $P<0.05$.

$\ddagger$ Also belongs to EURODIAB Ace Study.

SWithout Sardinia.

in girls, and in Cuba a decrease of $7.4 \%(3.5-11.3 \%)$ in boys and a decrease of $13.9 \%(10.3-17.4 \%)$ in girls.

In the pooled data, the increase was less in the older age groups: $4.0 \%(3.1-4.9 \%)$ in the $0-4$-year age group, $3.0 \%$ $(2.4-3.7 \%)$ in the $5-9$-year age group and $2.1 \%(1.5-2.7 \%)$ in the 10-14-year age group. This decrease in the annual increase over the age groups was seen mainly in European populations.
The trend was also estimated by continents (Table 3 ). The statistically significant increasing trend was $4.0 \%(1.8-6.2 \%)$ in Asia, 3.2\% (2.7-3.6\%) in Europe and 5.3\% (3.3-7.3\%) in North America. In Central America and the West Indies, the trend was a significant decrease at $3.6 \%(2.2-5.0 \%)$. However, a decreasing trend in this area was seen only in Cuba where the decrease was $10.8 \%$ (Table 1 ). In general, the 
Table 2 Poisson regression analyses of the incidence of Type 1 diabetes in age groups by sex (pooled analyses for all centres)

\begin{tabular}{lcll}
\hline & Age group (years) & Risk ratio & $95 \%$ CI \\
\hline Boys* & $0-4$ & 1.00 & \\
& $5-9$ & 1.52 & $(1.47 ; 1.58)$ \\
& $10-14$ & 1.94 & $(1.87 ; 2.01)$ \\
Girls* & $0-4$ & 1.00 & \\
& $5-9$ & 1.72 & $(1.66 ; 1.79)$ \\
& $10-14$ & 1.93 & $(1.86 ; 2.01)$ \\
Total $\dagger$ & $0-4$ & 1.00 & \\
& $5-9$ & 1.62 & $(1.57 ; 1.66)$ \\
& $10-14$ & 1.94 & $(1.89 ; 1.98)$ \\
\hline
\end{tabular}

*Adjusted for centre.

$\ddagger$ Adjusted for centre and sex.

increasing trend was strongest in the centres with very high incidence $3.7 \%(3.1-4.2 \%)$ and in centres with intermediate incidence $3.5 \%(2.8-4.2 \%)$. In the centres with low and very low incidence, there were no significant increases in incidence rates over time.

When Poisson regression models were fitted to the dataset (Table 4), the simplest model showed that there were statistically significant differences in incidence between centres and age groups, but not between genders (Table 4, lines 2, 4 and $3)$. The overall annual increase in incidence was statistically significant (line 5) and the effect of age and gender on the incidence was not similar in all centres (lines 6, 7 and 12). The trends in incidence varied between the age groups (line 11) and between centres (line 9).

\section{Discussion}

The rising incidence of Type 1 diabetes is a global phenomenon. The incidence of Type 1 diabetes has increased by $2.8 \%$ per year during the years 1990-1999 worldwide. Thus, the results of this study confirm the previously reported trend of $2.5-3.0 \%$ annual increase in incidence since the early $1970 \mathrm{~s}$
$[8,10]$. The total population of children aged 14 years or under (740 million) from countries for which the incidence of Type 1 diabetes is estimated using a standardized study protocol covers $41 \%$ of the world's population aged 14 years or under. The global variation in incidence ( $>350$-fold) appears to be stable, although we were not able to estimate the global range for the whole 10-year study period, because incidence data from most low incidence countries were available for the first 5-year period only. There was also within-country variation in incidence in several countries. However, it has not been possible to investigate this variation further in this review.

The global increase in incidence of Type 1 diabetes may be partly explained by an improvement in case ascertainment. An underestimation is certainly inherent in all registration systems, but the problem was avoided in this study by measuring the degree of ascertainment on the basis of two or more data sources. In some countries collecting incidence data, secondary sources for ascertainment of cases are not available or are difficult to find. However, $81 \%$ of registries checked the degree of ascertainment using two independent data sources. Most of the information of the incidence of Type 1 diabetes is from regions with a high or intermediate incidence, mostly in Europe and North America, where several registries have been established since the mid-1980s or even before. The data from Asia, South America and Africa are still sparse. However, the incidence appears to be increasing in almost all populations worldwide and statistically significant increases were found in all continents except in South America, Central America and the West Indies. The latter was the only continent where there was a significantly decreasing trend in this study.

Unfortunately, information is lacking from many countries with very low gross domestic product and the largest child populations. These are mostly countries in Africa and South and East Asia, where infectious diseases are the greatest childhood killers. When one child in 10 dies before his/her fifth birthday, as happens in these countries today, it may be practically impossible to get reliable data on the incidence of diabetes among children. The symptoms of diabetes surely remain

Table 3 Annual increase (\%) of incidence of Type 1 diabetes by age groups and by continents in children aged 14 or under in 1990-1999; the results of the Poisson regression analyses

\begin{tabular}{|c|c|c|c|c|c|c|c|c|c|c|}
\hline \multirow[b]{2}{*}{ Continent } & \multicolumn{3}{|c|}{ Total Age/ year* } & \multicolumn{2}{|l|}{$0-4$ years } & \multicolumn{2}{|l|}{$5-9$ years } & \multicolumn{2}{|c|}{$10-14$ years } & \multirow[b]{2}{*}{$P$} \\
\hline & Trend \% & $95 \% \mathrm{CI}$ & $P$ & Trend \% & $95 \% \mathrm{CI}$ & Trend \% & $95 \%$ CI & Trend \% & $95 \% \mathrm{CI}$ & \\
\hline Africa & 3.0 & $(0.3 ; 5.8)$ & 0.029 & 0.9 & $(-5.6 ; 7.9)$ & 9.2 & $(3.8 ; 14.9)$ & 0.6 & $(-3.0 ; 4.3)$ & 0.154 \\
\hline Asia & 4.0 & $(1.8 ; 6.2)$ & $<0.001$ & 1.3 & $(-3.5 ; 6.2)$ & 5.0 & $(1.4 ; 8.8)$ & 5.1 & $(1.8 ; 8.5)$ & 0.238 \\
\hline Europe & 3.2 & $(2.7 ; 3.6)$ & $<0.001$ & 4.7 & $(3.9 ; 5.6)$ & 3.4 & $(2.7 ; 4.0)$ & 2.2 & $(1.6 ; 2.8)$ & $<0.001$ \\
\hline North America & 5.3 & $(3.3 ; 7.3)$ & $<0.001$ & 6.9 & $(2.4 ; 11.5)$ & 4.7 & $(1.4 ; 8.1)$ & 5.1 & $(2.3 ; 8.1)$ & 0.674 \\
\hline South America & 5.3 & $(2.8 ; 7.9)$ & $<0.001$ & 6.5 & $(1.2 ; 12.0)$ & 9.2 & $(4.7 ; 13.9)$ & 1.7 & $(-2.0 ; 5.6)$ & 0.071 \\
\hline Central America and West Indies & -3.6 & $(-5.0 ;-2.2)$ & $<0.001$ & -3.6 & $(-6.7 ; 0.3)$ & -4.2 & $(-6.5 ;-1.9)$ & -3.2 & $(-5.3 ; 1.0)$ & 0.881 \\
\hline Oceania & 3.2 & $(-0.4 ; 6.9)$ & $<0.001$ & 11.1 & $(3.2 ; 19.6)$ & -0.6 & $(-6.4 ; 5.5)$ & 2.1 & $(-3.3 ; 7.8)$ & 0.042 \\
\hline All regions & 2.8 & $(2.4 ; 3.2)$ & $<0.001$ & 4.0 & $(3.1 ; 4.9)$ & 3.0 & $(2.4 ; 3.7)$ & 2.1 & $(1.5 ; 2.7)$ & $<0.001$ \\
\hline
\end{tabular}

"Interaction between age group and calendar year. 
Table 4 Summary of the Poisson regression analyses of the incidence of Type 1 diabetes for data from 105 populations worldwide during the period 1990-1999

\begin{tabular}{|c|c|c|c|c|c|c|}
\hline \multirow[b]{2}{*}{ Model terms* } & & \multicolumn{2}{|c|}{ Suitability of fit } & \multicolumn{3}{|c|}{ Test for last term } \\
\hline & & Deviance & d.f. & Deviance & d.f. & $P$-value \\
\hline 1 & Constant & 57767.7 & 4493 & & & \\
\hline 2 & $(1)+$ population & 8595.9 & 4389 & 49171.8 & 104 & $<0.0001$ \\
\hline 3 & $(2)+\operatorname{sex}$ & 8594.0 & 4388 & 1.9 & 1 & 0.171 \\
\hline 4 & $(3)+$ age & 5713.4 & 4386 & 2880.6 & 2 & $<0.0001$ \\
\hline 5 & $(4)+$ year & 5472.1 & 4385 & 241.4 & 1 & $<0.0001$ \\
\hline 6 & $(5)+$ population/sex & 5279.6 & 4281 & 192.4 & 104 & $<0.0001$ \\
\hline 7 & $(6)+$ population/age & 4703.7 & 4073 & 576.0 & 208 & $<0.0001$ \\
\hline 8 & (7) + sex/age & 4663.7 & 4071 & 40.0 & 2 & $<0.0001$ \\
\hline 9 & (8) + population/year & 4325.4 & 3967 & 338.2 & 104 & $<0.0001$ \\
\hline 10 & (9) + sex/year & 4323.3 & 3966 & 2.1 & 1 & 0.145 \\
\hline 11 & $(10)+$ age/year & 4303.9 & 3964 & 19.4 & 2 & $<0.0001$ \\
\hline 12 & $(11)+$ population/sex/age & 4070.6 & 3756 & 233.3 & 208 & 0.126 \\
\hline 13 & (12) + population/sex/year & 3969.2 & 3652 & 101.4 & 104 & 0.565 \\
\hline 14 & $(13)+$ population/age/year & 3709.0 & 3444 & 260.1 & 208 & 0.011 \\
\hline 15 & $(14)+$ sex/age/year & 3704.3 & 3442 & 4.8 & 2 & 0.091 \\
\hline 16 & $(15)+$ population/sex/age/year & 3475.5 & 3234 & 228.7 & 208 & 0.154 \\
\hline
\end{tabular}

"Population = centre; sex = gender; age = variable categorized into three groups 0-4, 5-9 and 10-14 years; year = calendar year.

unrecognized when children die, for example, from acute respiratory infections or diarrhoea. Thus, reliable estimation of the incidence of diabetes in these areas may be possible only after child mortality decreases.

The risk of Type 1 diabetes has been increasing since the 1950s and there are no signs that this trend is abating $[8,10,13]$. The results of this analysis confirm the widely believed assumption that the increase in the incidence of Type 1 diabetes has been very rapid, particularly in the youngest age groups in European populations [14-18]. Another widely adopted assumption that high incidence regions, except Finland, would have reached a plateau is not supported by our analysis. We found no significant differences in the trend of incidence levels over time between participating countries.

The constantly increasing incidence of Type 1 diabetes over such a short period of time (10 years) cannot be explained by shifts in genetic susceptibility alone. Hence, causative agents should be sought in the environment or in gene-environment interaction. There is already some evidence that rapid growth and obesity in early childhood increases the risk of Type 1 diabetes [19]. Further, psychological stress in families has been found to be associated with diabetes-associated autoantibodies in 1-year-old children [20]. Recent studies [21-24] have shown that environmental factors have a stronger effect on genetically non-susceptible individuals than those genetically susceptible to getting Type 1 diabetes. It might be timely to extend the study of the environmental agents to completely new areas of children's lifestyle, including social circumstances, stressful life events, and health behaviour in the widest sense.

In conclusion, the incidence of childhood Type 1 diabetes continues to rise and continuous monitoring, using updated diagnostic criteria and developed standardized methods, is required. In particular, it is important to differentiate between Type 1 and Type 2 diabetes, because the latter has become increasingly common in adolescents in some populations The fact that prevention strategies are presently in progress justifies ongoing surveillance of incidence: i.e. when these trials are performed on a population basis, surveillance will allow us to demonstrate their effectiveness.

\section{Competing interests}

None declared.

\section{Acknowledgements}

This work was supported by grants from Academy of Finland, project no. 41226 and project no. 207008. We would like to thank the EURODIAB Study Group and the project coordinators Professor Anders Green and Professor Gyula Soltesz for the collaborative support to this study.

\section{References}

1 WHO DIAMOND Project Group. WHO Multinational Project for Childhood Diabetes. Diabetes Care 1990; 13: 1062-1068.

2 Karvonen M, Viik-Kajander M, Moltchanova E, Libman I, LaPorte R, Tuomilehto J for the Diabetes Mondiale (DIAMOND) Project Group. Incidence of the childhood Type 1 diabetes worldwide. Diabetes Care 2000; 23: 1516-1526.

3 Diabetes Epidemiology Research International Group. Geographic patterns of childhood insulin-dependent diabetes mellitus. Diabetes 1988; 37: 1113-1119. 
4 Diabetes Epidemiology Research International Group. Secular trends in incidence of childhood IDDM in 10 countries. Diabetes 1990; 39: 858-864.

5 Rewers M, LaPorte RE, King H, Tuomilehto J, DERI Group. Trends in the prevalence and incidence of diabetes: insulin-dependent diabetes mellitus in childhood. World Health Stat Quarterly 1990; 41: 179-189.

6 Green A, Gale EA, Patterson CC. Incidence of childhood-onset insulin-dependent diabetes mellitus: the EURODIAB ACE Study. Lancet 1992; 339: 905-909.

7 Karvonen M, Tuomilehto J, Libman I, LaPorte R. A review of the recent epidemiological data on the worldwide incidence of type 1 (insulin-dependent) diabetes mellitus. World Health Organization DIAMOND Project Group. Diabetologia 1993; 36: 883-892.

8 Green A, Patterson CC on the behalf of the EURODIAB TIGER Study Group. Trends in the incidence of childhood-onset diabetes in Europe 1989-1998. Diabetologia 2001; 44: B3-B8.

9 Rytkönen M, Ranta J, Tuomilehto J, Karvonen M, The SPAT Study Group, The Finnish Childhood Diabetes Registry Group. Bayesian analysis of geographical variation in the incidence of Type I diabetes in Finland. Diabetologia 2001; 44: B 37-B44.

10 Onkamo P, Väänänen S, Karvonen M, Tuomilehto J. Worldwide increase in incidence of Type I diabetes - the analysis of the data on published incidence trends. Diabetologia 1999; 42: 1395-1403.

11 World Health Organization. Diabetes Mellitus. Report of WHO Study Group, Technical Report Series no. 727. Geneva: World Health Organization, 1985.

12 LaPorte RE, McCarty D, Bruno G, Tajima N, Baba S. Counting diabetes in the next millennium. Application of capture-recapture technology. Diabetes Care 1993; 16: 528-534.

13 Gale EAM. The rise of childhood type 1 diabetes in the 20th century. Diabetes 2002; 51: 3353-3361.

14 Karvonen M, Pitkäniemi J, Tuomilehto J, the Finnish Childhood Diabetes Registry Group. The onset age of Type 1 diabetes in Finnish children has become younger. Diabetes Care 1999; 22: 1066-1070.

15 Ruwaard D, Gijsen R, Bartelds AIM, Hirasing R, Verkleij H, Kromhout $\mathrm{D}$. Is the incidence of diabetes increasing in all age groups in the Netherlands? Diabetes Care 1996; 19: 214-218.

16 Dahlquist G, Mustonen L. Childhood onset diabetes time trends and climatological factors. Int J Epidemiol 1994; 23: 1234-1241.

17 Schober E, Schneider E, Waldhör T, Tuomilehto J, Austrian Diabetes Incidence Study Group. Increasing incidence of IDDM in Austrian children. A nationwide study in 1979. Diabetes Care 1995; 18: 1280-1283.

18 Gardner SG, Bingley PJ, Satwell PA, Weeks S, Gale EM, the Bart's-Oxford Study Group. Rising incidence of insulin-dependent diabetes in children aged under 5 years in the Oxford region: time trend analysis. Br Med J 1997; 315: 713-717.

19 Hyppönen E, Virtanen SM, Kenward MG, Knip M, Åkerblom HK, the Childhood Diabetes in Finland Study Group. Obesity, increased linear growth, and risk of type 1 diabetes in children. Diabetes Care 2000; 23: 1755-1760.

20 Sepa A, Wahlberg J, Vaarala O, Frodi A, Ludvigsson J. Psychological stress may induce diabetes-related autoimmunity in infancy. Diabetes Care 2005; 28: 290-295.

21 Hermann R, Knip M, Veijola R, Simell O, Laine A-P, Åkerblom HK et al., the FinnDiane Study Group. Temporal changes in the frequencies of HLA genotypes in patients with type 1 diabetes - indication of an increased environmental pressure? Diabetologia 2003; 46: $420-425$.

22 Kaila B, Dean HJ, Schroeder M, Taback SP. HLA, day care attendance, and socio-economic status in young patients with type 1 diabetes. Diabet Med 2003; 20: 777-780.

23 Gillespie KM, Bain SC, Barnett AH, Bingley PJ, Christie MR, Gill GV. The rising incidence of childhood type 1 diabetes and reduced contribution of high-risk HLA haplotypes. Lancet 2004; 364: 1699 1700 .
24 Carle F, Gesuita R, Bruno G, Coppa GV, Falorni A, Lorini R et al., the RIDI Study Group. Diabetes incidence in 0- to 14-year age group in Italy: a 10-year prospective study. Diabetes Care 2004; 27: 2790-2796.

\section{Appendix}

\section{WHO Multinational Project for Childhood Diabetes (DIAMOND) Research Group:}

Algeria: Dr K. Bessaoud, Dr G. Boudraa (Oran); Argentina: Dr M. Molinero de Ropolo (Cordoba), Dr M. de Sereday, M. L. Marti, Dr M. Moser (Avellaneda), Dr S. Lapertosa (Corrientes), Dr M. Damiano (Tierra del Fuego); Australia: Dr C. Verge, Dr N. Howard (NSW); Austria: Dr E. Schober; Barbados: Dr O. Jordan; Belgium: Dr I. Weets, Dr F. Gorus, Dr M. Coeckelberghs, Dr R. Rooman, Dr L. Van Gaal (Antwerp region); Brazil: Dr L. J. Franco, Dr S. R. G. Ferreira (3 centres, Sao Paulo state), Dr H. P. K. Lisbóa, Dr L. A. Kurtz, R. Graebin, L. Kutzke, C. Rodriges (Passo Fundo); Bulgaria: Dr R. Savova, Dr V. Christov (West-Bulgaria), Dr V. Iotova, Dr V. Tzaneva (Varna); Canada: Dr D. Pacaud (Calgary), Dr E. Toth (Edmonton), Dr M. H. Tan (Prince Edward Island); Chile: Dr E. Carrasco, Dr F. Pérez (Santiago); China: Dr Y. Ze (Henan, Dalian, Guilin, Jilin, Nanning, Zunyi), Dr Y. Bo (Tieling), Dr S. Chen, Dr L. Fu (Jinan), Dr L. Deng (Sichuan), Dr S. Shen (Shanghai), Dr K. Teng (Wulumuqi), Dr C. Wang, Dr H. Jian, Dr J. Ju (Zhengzhou), Dr C. Yan, Dr Y. Ze (Beijing), Dr Y. Deng, Dr C. Li (Changchun), Dr Y. Zhang (Jilin province) Dr Y. Liu, Dr X. Long (Shenyang), Dr Z.Zhen (Huhehot), Dr Z. Sun (Dalian), Dr B. Wang (Harbin), Dr G. Wong (Hong Kong); Colombia: Dr O. V. Orrego (Cali), Dr P. Aschner (Bogotà, D.C.); Cuba: Dr O. Diaz-Diaz, Dr O. Mateo de Acosta; Czech Republic: Dr O. Cinek, Dr J. Vavrinec; Denmark: Dr B. S. Olsen, Dr A. J. Svendsen, Dr J. Kreutzfeldt, Dr E. Lund (4 counties); Dominica: Dr E. S. Tull; Dominican Republic: Dr A. Selman-Geara, Dr A. S. Almonte; Estonia: Dr T. Podar; Finland: Dr J. Tuomilehto, Dr M. Karvonen, Dr I.-L. Notkola, Dr E. Moltchanova, O. Taskinen; France: Dr C. Levy-Marchal, Dr P. Czernichow (4 regions); FYR of Macedonia: Dr M. Kocava; Germany: Dr A. Neu, Dr S. Ehehalt (Baden-Württemberg), Dr J. Rosenbauer, Dr G. Giani, Dr A. Icks (Governmental district of Düsseldorf, 7 counties); Greece: Dr C. Bartsocas, Dr A. Vazeou (Attica region); Hungary: Dr G. Soltesz (18 counties); Israel: Dr Z. Laron, Dr O. Gordon, Dr Y. Albag, Dr I. Shamis; Italy: Dr F. Purrello, Dr M. Arpi, Dr G. Fichera, Dr M. Mancuso, Dr C. Lucenti (Eastern Sicily), Dr G. Chiumello (Lombardia region), Dr G. Bruno, Dr G. Pagano (Turin province), Dr M. Songini, Dr A. Casu, Dr A. Marinaro, Dr P. Frongia, Dr M. A. Zedda, Dr A. Milia (Sardinia), Dr M. Tenconi, Dr G. Devoti (Pavia province), Dr P. Pozzilli. Dr N. Visalli, Dr L. Sebastiani, Dr G. Marietti, Dr R. Buzzetti (Lazio region), Dr V. Cherubini (Region Marche); Japan: Dr A. Okuno, Dr S. Harada, Dr N. Matsuura (Hokkaido), Dr E. Miki, Dr S. Miyamoto, Dr N. Sasaki (Chiba), Dr G. Mimura (Okinawa); Kuwait: Dr A. Shaltout, 
Dr M. Qabazrd, Dr M. Al-Khawari; Latvia: Dr G. Brigis, Dr I. Dzivite; Libya: Dr O. Kadiki (Benghazi); Lithuania: Dr B. Urbonaite; Luxembourg: Dr C. de Beaufort; Mauritius: Dr H. Gareeboo; Mexico: Dr O. Aude Rueda (Veracruz); the Netherlands: Dr M. Reeser (5 regions); New Zealand: Dr R. Elliott (Auckland), Dr R. Scott, Dr J. Willis, Dr B. Darlow (Canterbury); Norway: Dr G. Joner (8 counties); Pakistan: Dr G. Rafique, Dr F. Jawad (Karachi); Paraguay: Dr J. Jimenez, Dr C. M. Palacios, Dr F. Cañete, Dr J. Vera, Dr R. Almiron. G. E. Benítez, C. S. Espínola; Peru: Dr S. Seclén (Lima); Poland: Dr D. Woznicka (Wielkopolska), Dr Z. Szybinski, Dr E. Placzkiewicz (Warsaw, Cracow, Wroclaw), Dr P. Jarosz-Chobot (Upper Silesia region); Portugal: Dr C. Menezes (Portalegre), Dr E. A. Pina (Algarve region), Dr M. M. A. Ruas, Dr F. J. C. Rodrigues (Coimbra), Dr S. Abreu (Madeira Island); Romania: Dr C. Ionescu-Tirgoviste (Bucharest region); Russia: Dr E. Shubnikov (Novosibirsk); Slovakia: Dr D. Michalkova, Dr L.
Bark, Dr P. Hlava, Dr M. Mikulecky; Slovenia: Dr C. Krzisnik, Dr T. Battelino, Dr N. Bratina Ursic, Dr P. Kotnik; South Korea: Dr K. W. Ko (Seoul); Spain: Dr C. Castell, Dr A. Goday (Catalonia); Sudan: Dr M. Magzoub (Gezira province); Sweden: Dr G. Dahlquist; Switzerland: Dr E. Schoenle; Tunisia: Dr K. Nagati (Kairouan), Dr F. B. Khalifa (Gafsa, Beja, Monastir); UK: Dr A. Burden, N. Raymond (Leicestershire), Dr B. A. Millward, Dr H. Zhao (Plymouth), Dr C. C. Patterson, Dr D. Carson, Dr D. Hadden (Northern Ireland), Dr P. Smail, Dr B. McSporran, Dr N. Waugh (Scotland), Dr P. Bingley (Oxford region), Dr P. A. McKinney, Dr R. G. Feltbower, Dr H. J. Bodansky, Dr F. Campbell (Yorkshire); USA: Dr E. S. Tull (Virgin Islands), Dr R. E. LaPorte, Dr I. Libman (Allegheny County, PA), Dr J. Roseman, Dr S.M. Atiquar Rahman (Jefferson county, AL), Dr T. Frazer de Llado (Puerto Rico), Dr R. Lipton (Chicago, IL); Uruguay: Dr A. M. Jorge (Montevideo); Venezuela: Dr P. Gunczler, Dr R. Lanes (Caracas 2). 
Copyright of Diabetic Medicine is the property of Blackwell Publishing Limited and its content may not be copied or emailed to multiple sites or posted to a listserv without the copyright holder's express written permission. However, users may print, download, or email articles for individual use. 